Neuromuscular Disorders

\title{
Results of an open label feasibility study of Sodium Valproate in people with McArdle disease
}

--Manuscript Draft--

\begin{tabular}{|c|c|}
\hline Manuscript Number: & NMD-D-20-00030R1 \\
\hline Keywords: & $\begin{array}{l}\text { Glycogen Storage Disease type V; valproic acid (VPA); VO2peak; 12-minute walking } \\
\text { test; Outcome measures }\end{array}$ \\
\hline Corresponding Author: & $\begin{array}{l}\text { Ros Quinlivan, MBBS, MD } \\
\text { University College London Hospitals NHS Foundation Trust } \\
\text { London, United Kingdom }\end{array}$ \\
\hline First Author: & Renata S Scalco, MD, PhD \\
\hline \multirow[t]{10}{*}{ Order of Authors: } & Renata S Scalco, MD, PhD \\
\hline & Mads Stemmerik, MD \\
\hline & Nicoline Løkken, MD \\
\hline & Zuzanna Michalak, PhD \\
\hline & Jatin Pattni, PhD \\
\hline & Richard Godfrey, PhD \\
\hline & George Samandouras, FRCS \\
\hline & Paul Bassett, MSc. \\
\hline & Janice L. Holton, PhD \\
\hline & Ronald G Haller, MD \\
\hline Suggested Reviewers: & Alejandro Lucia \\
\hline
\end{tabular}


Professor, Universidad Europea de Madrid

alejandro.lucia@universidadeuropea.es

experienced in designing and running trials in GSDs and using exercise tests

Nicol Voermans

Radboud Universiteit

Nicol.Voermans@radboudumc.nl

Experienced in GSDs and clinical trials

Tomas Pinos

Vall d'Hebron Institut de Recerca

tomas.pinos@vhir.org

Published the animal model studies of valproate in GSDV, which inspired this clinical trial

Response to Reviewers: 
$20^{\text {th }}$ April 2020

Dear Victor

Re: Results of an open label feasibility study of sodium Valproate in people with McArdle disease

Thank you very much for giving us an opportunity to re-submit a revised manuscript. We are very grateful to your comments and those of the peer reviewers

With best wishes

Ros 


\section{SUBMISSION CHECKLIST for Neuromuscular Disorders}

Please ensure that your paper conforms to the following guidelines. Once you have completed the checks, please upload this file as a separate document using the file type "Checklist".

Title: No abbreviations are to appear in the title

\section{Authors' names}

\section{Authors' affiliations/addresses}

Address (including fax number and e-mail address) of the corresponding author

\section{Abstract:}

a max. 200 words (150 for Case Reports)

- No abbreviations to appear in the abstract

․ Continuous text with no sub-headings

Pages: numbered

\section{Keywords}

Highlights: $3-5$ bullet points (each to be no more than 85 characters including spaces)

Font size 12pt

Single-column printout

Double-spaced text

Language:

$\square$ Spelling checked

$\square$ Grammar checked

Figures: $\square$ Acceptable line quality and must fit on a portrait page

$\square$ Uploaded as separate files not embedded in the text

$\square$ Colour figures must clearly marked as being intended for colour reproduction

Consent for recognisable figures provided (if applicable) and uploaded with the manuscript files

Figure and Table legends: Separate list provided

Tables:

$\square$ Should be in portrait format, but if landscape they must fit across a portrait page

$\square$ Uploaded as separate files and not embedded in the text

References: For six or more authors, please list the first six names followed by "et al."

\section{Author Agreement:}

It is the corresponding author's responsibility to ensure all co-authors have read and agreed the contents of the paper (on initial submission and on any revisions or subsequent resubmissions). Please prepare a statement confirming that all authors have agreed the contents of the submission. Please include the title and date, and signature. The corresponding author may sign on behalf of all coauthors. 
$16^{\text {th }}$ April 2020

Dear Victor,

Re: Results of an open label feasibility study of sodium Valproate in people with McArdle Disease

Thank you very much for giving us an opportunity to submit a revised manuscript following peer review. We are very grateful to yourself and the peer reviewers for their helpful comments. I have re-submitted a revised manuscript with track changes. Our responses to the specific comments are also highlighted below in red.

With best wishes

Ros

\section{Editor's comment}

This phase II, open label, feasibility pilot study to assess efficacy of six months treatment with VPA ( $20 \mathrm{mg} / \mathrm{kg} / \mathrm{day}$ ) included 16 people with McArdle disease..... showed no benefit.

Guess it is a matter of semantics, but I always looked upon open label preliminary studies as preliminary studies from which one could not draw definitive conclusions. But perhaps an open label study that is negative is enough to decide not to proceed further.......

Thanks for this comment, we have removed any statement indicating a definitive outcome and have amended the text in the conclusion to read as follows: 'This feasibility study to assess efficacy of 20 $\mathrm{mg} / \mathrm{kg} /$ day VPA in McArdle disease was planned to power a larger placebo-controlled trial of VPA in this patient population. Our results demonstrated that VPA was well-tolerated but there was no clinically meaningful benefit after 6 months of treatment.'

\section{Reviewer \#1:}

Results of an open label feasibility study of Sodium Valproate in people with McArdle disease

General comment

* Well written

Abstract

* Clear and concise

* Could a few comments about the secondary outcome measures be included?

We have added some comments about secondary outcomes in the abstract

Introduction: 
Results

* 17 recruited (11 men and 5 women) is not correct ( $11=5=16$, not 17)

Thank you for spotting this typo, the number of participants has been corrected: 14 completed, 2 dropped out, one withdrawn

Discussion:

* It would be better to start with a summary of the main findings and subsequently discuss these findings in light of the studies in animals

We have added a paragraph regarding the main findings

* It would be nice if the authors could discuss the reasons why they think that VPA did not exert the expected effect in some more detail

We do not know why VPA did not have the expected positive outcome, we have added a paragraph describing other conditions SMA and ALS where a similar effect was found i.e. positive animal studies but negative clinical trials. We have also made the point in our discussion that VPA was administered intramuscularly in one animal study which could have resulted in muscle damage and then phosphorylase re-expression

* Could the authors add which alternative HDAC agents could be used in future trials?

We have added the following paragraph 'VPA and phenylbutyrate are classed as Aliphatic HDAC inhibitors, it's possible that this group of drugs have less of an effect on skeletal muscle or anterior horn cell in humans. There are other classes of HDAC inhibitors, although they have greater toxicity and are usually used for cancer treatment for example Vorinostat, Romidepsin, Panobinostat, and Belinostat. The unwanted adverse effects may mean that these drugs are less likely to be suitable for people with neuromuscular disease.'

* Did the results in this study lead to any other new insights?

We have added a sentence explaining that VPA was tolerated in McArdle disease, therefore it can be used safely for other indications such as epilepsy, migraine and bipolar disorder in this population.

* Was the weight gain more than reported in cohorts of patients with epilepsy treated with VPA? Were patients warned for this?

No it was not worse than expected, we have added a sentence to this effect

\section{Reviewer \#2:}

This is a necessary and important work that properly analyzes the effects of VPA in McArdle disease patients. The manuscript is well written, properly structured and organised and results are clear. However, I feel that previous its publication some minor issues should be addressed:

1) In the introduction section it is stated that "In mature skeletal muscle fibres BGP expression is suppressed due to methylation of the BGP gene promoters as part of a post-natal downregulation of this foetal enzyme (references 13-15)". In this regard, as far as this reviewer is concerned, it has never been experimentally proven that Pygb and/or Pygl gene promoters are methylated, and that this methylation is causing the down regulation of Pygb/Pygl expression postnatally. It is true that different genome browsers, such as UCSC genome browser, shows that Pygb and Pygl promoters 
present Cpg islands, but whether these are methylated or not, and whether these methylations cause a downregulation of Pygb and/or Pygl gene expression postnatally has not yet been proven. It is very likely that methylation of these promoters occur, but it can not be stated in the introduction as a proven fact. Besides, references 13 to 15 do not support the statement.

This was a hypothesis, we have removed the statement

2) In the highlights the abreviation for sodium valproate is VS while throughout the manuscript is VPA. In keywords the term valproic acid is used, while in the manuscript sodium valproate is used instead.

We have changed to VPA throughout

3) A little explanation of why the VPA dose of $20 \mathrm{mg} / \mathrm{kg} / \mathrm{day}$ and also the length of the treatment were chosen would be useful.

We have added the following statement in the methods section 'This dose was chosen as it is the lowest recommended dose for treating epilepsy and we wanted to minimise known drug-related side-effects such as weight gain, drowsiness and thrombocytopenia that might outweigh any potential benefit. The daily dose was rounded up to the nearest available tablet strength and the maximum dose was $2 \mathrm{~g} /$ day. Treatment for six months was chosen as a cut-off point for the end of the trial, the ovine clinical trial was conducted over 15 weeks and showed the number of phosphorylase positive fibres increased over time ${ }^{17}$. We, therefore, decided to treat our patients for a longer time period to maximise any potential positive impact.'

4) Although the authors find in McA patient biopsies some percentage of regenerating fibres, these are not positive for glycogen phosphorylase. In the introduction is stated that regenerating fibres express glycogen phosphorylase (potentially Pygb or Pygl isoforms). Isn't it surprising? Any possible explanation? Any positive control for the technique was used?

A positive control was used and the stain was positive on smooth muscle fibres of vessels, it is possible that the fibres that were positive for neonatal myosin were not regenerating fibres. We have added a statement and very recent reference to that effect.

5) If the hypothesis is that VPA enhances the expression of the Pygb and/or Pygl isoforms, did the authors ever considered the possibility to analyse Pygb/Pygl mRNA by qPCR or protein levels by western blot?. Please, develop why these techniques were not used.

We have made some edits to the text and added a reference

'The histochemical method used identifies all isoforms of phosphorylase, given that the mutations in PYGM result in no phosphorylase expression, it was not considered necessary to include other methods to identify specific phosphorylase isoforms such as PCR.'

'Although neonatal myosin is often used as a marker for fibre regeneration there is evidence it can be up-regulated ${ }^{20}$. Muscle biopsies from a variety of neuromuscular conditions often show fibres with neonatal myosin for unknown reasons ${ }^{14}$. In our study the presence of a few fibres that showed neonatal myosin but no phosphorylase suggests that these were not regenerating fibres but rather that neonatal myosin had been upregulated. There are currently no antibodies to the brain isoform that reliably work on human muscle biopsies, however, the histochemical stain for phosphorylase detects all three isoforms of phosphorylase, we are therefore confident that VPA treatment did not up-regulate either the brain or liver isoform in our participants. In the sheep study ${ }^{17}$, it was not possible to completely exclude a local toxic effect of intramuscular VPA, which 
could have triggered muscle regeneration and thus the expression of foetal isozyme in injected muscles. Saline injected sheep showed a few fibres with neonatal myosin suggesting that mild muscle damage may have resulted from injection. In addition, there was a mild inflammatory response which was not seen in the muscle biopsies of our participants treated with oral VPA. An in vitro study analysed muscle cultures from KI mice exposed to VPA for 72 hours at 1,2 and $5 \mathrm{mM}$ and showed a dose-dependent increase in BGP was shown together with a reduction in intracellular glycogen content ${ }^{18}$

Reference: V Dubowitz, C Sewry, A Oldfors. Metabolic Myopathies 1: Glycogenoses and Lysosomal Myopathies. In: Muscle Biopsy: A Practical Approach. Fifth Edition 2020, Elsevier Oxford 
Highlights

- Sodium Valproate (VS) stimulates expression of BGP in GSDV animal models

- An open label study of VS in GSDV showed no benefit in people with GSDV

- VS did not stimulate the expression of BGP in people with GSDV 
Results of an open label feasibility study of Sodium Valproate in people with

\section{McArdle disease}

Renata S Scalco, MD, PhD a,b, Mads Stemmerik, MDc, Nicoline Løkken, MDc,

Christoffer R. Vissing, $\mathrm{MD}^{\mathrm{c}}$, Karen L. Madsen, $\mathrm{MD}^{\mathrm{c}}$, Zuzanna Michalak, PhDa, Jatin Pattni, PhDa, Richard Godfrey, PhD a,d, George Samandouras, FRCS ${ }^{a}$, Paul Bassett, MSc. ${ }^{\mathrm{e}}$, Janice L. Holton, $\mathrm{PhD}^{\mathrm{a}}$,--Thomas Krag, MDc, Ronald G Haller, MD ${ }^{\dagger}$, , C Sewry $\underline{P h D g}$, John Vissing, $\mathrm{PhD}^{\mathrm{c}}$, Ros Quinlivan, MBBS, MDa

a) UCL Institute of Neurology and National Hospital for Neurology and Neurosurgery, Queen Square, UK

b) CAPES Foundation, Ministry of Education, Brazil

c) Copenhagen Neuromuscular Center, Department of Neurology, Rigshospitalet, University of Copenhagen, Denmark

d) Centre for Human Performance, Exercise and Rehabilitation, Brunel University London, Uxbridge, UK

e) Statsconsultancy Ltd., HP7 9EN, UK

f) Department of Neurology, The University of Texas Southwestern Medical Center, Dallas, USA and Neuromuscular Centre, Institute for exercise and environmental medicine

f) g) RJAH Orthopaedic Hospital NHS Foundation Trust Robert Jones and Agnes Hunt NHD Foundation NHS Trust, Oswestry, UK
Commented [CS1]:

Formatted: Not Superscript/ Subscript

\section{Corresponding Author:}

Dr Ros Quinlivan 


\section{r.quinlivan@ucl.ac.uk}

8-11 Queen Square, WC1N 3BG, London, UK

\section{$+442034488013$}

The corresponding author ensures all co-authors have read and agreed the contents of the paper

\section{ABSTRACT}

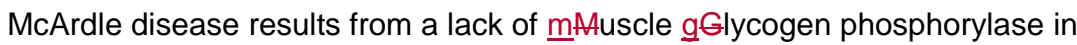
skeletal muscle tissue. Regenerating skeletal muscle fibres can express the brain glycogen phosphorylase isoenzyme. Stimulating expression of this enzyme could be a therapeutic strategy. Animal model studies indicate that sodium valproate (VPA) can increase expression of phosphorylase in skeletal muscle affected with McArdle disease. This study was designed to assess whether VPA can modify expression of brain phosphorylase isoenzyme in people with McArdle disease. This phase II, open label, feasibility pilot study to assess efficacy of six months treatment with VPA (20 $\mathrm{mg} / \mathrm{kg} / \mathrm{day}$ ) included 16 people with McArdle disease. Primary outcome assessed changes in $\mathrm{VO}_{2}$ peak during an incremental cycle test. Secondary outcomes included: phosphorylase enzyme expression in post-treatment muscle biopsy, total walked-distance walked in 12 minutes, plasma lactate change (forearm exercise test) and quality of life (SF36). Safety parameters were collected. 14 participants completed the trial, VPA treatment was well tolerated; weight gain was the most frequently reported drug-related adverse event. There was no clinically meaningful change in any ofeither the primary or secondary outcome measures including: $\mathrm{VO}_{2}$ peak, 12 minute walk test and muscle biopsy to look for a change in the number of phosphorylase positive fibres -between baseline and 6 months of treatment. 
Although this was a small open label feasibility study, it suggests that a larger

randomised controlled study of VPA, may not be worthwhile. or any other secondary outcomes. Treatment with VPA does not benefit people with McArdle diseasestudy participants.

\section{KEYWORDS:}

Glycogen Storage Disease type V, Sodium Valproate (VPA) valproic acid, $\mathrm{VO}_{2}$ peak, 12-minute walking test, outcome measures

\section{INTRODUCTION:}

McArdle disease is an autosomal recessive condition caused by mutations in the muscle glycogen phosphorylase gene (PYGM). Affected patients lack the enzyme, muscle glycogen phosphorylase (MGP), which is essential for glycogen breakdown in skeletal muscles ${ }_{2^{-}}{ }^{1-4}$ whichThis results in severe impairment of physical activity, especially when the onset of exercise is abrupt, high intensity or isometric in nature.,$\frac{5,65-8}{6}$ Currently, $y$, there is no satisfactory drug treatment for McArdle disease. Identifying new therapeutic strategies are therefore warranted. ${ }^{9-7}$

Mammals have three glycogen phosphorylase isoforms encoded by different genes that are tissue specific: muscle (MGP), liver (LGP) and brain (BGP). $\frac{8-1010-12}{\mathrm{~L}}$ MGP, the exclusive form expressed in mature skeletal muscle fibres, is absent in people with McArdle disease due to recessively inherited mutations in the corresponding gene. $\cdot{ }^{910} \mathrm{BGP}$ is encoded by $P Y G B$ and is expressed in developingfoetal muscle tissue both in vivo and in vitro, and it is thus transientlymay be temporarily expressed in regenerating skeletal muscle fibres. ${ }^{13-1511-14}$ In mature
Commented [SR $\{2]$ : This key word was added as an extra way to search for our manuscript, and that's the role of the key words, to provide alternative word. Please find below the reviewer comment:

(I suggest to amend the highlights to match VPA, and to keep the key words and manuscript the way it is):

In the highlights the abreviation for sodium valproate is VS while throughout the manuscript is VPA. In keywords the term valproic acid is used, while in the manuscript sodium valproate is used instead.

Formatted: Font: (Default) Arial, Bold

Formatted: Normal, No bullets or numbering

Formatted: Superscript

Formatted: Superscript

Formatted: Superscript

Formatted: Font: Italic

Formatted: Font: Italic

Formatted: Superscript 
skeletal muscle fibres BGP expression is suppressed due to methylation of the BGP gene promoters as part of a post-natal downregulation of this foetal enzyme ${ }^{13-15}$ -

In-vitro studies on human primary skeletal muscle cell cultures derived from people with McArdle disease showed expression of BGP. ${ }^{13}$ Such findings combined with knowledge of the normal physiological response to muscle damage (muscle regeneration) suggest that pharmaceutical reactivation of BGP in mature skeletal muscle fibres may be a therapeutic strategy for McArdle disease..$^{13}$

Sodium valproate (Valproic Acid, VPA) belongs to a group of drugs known as 'histone deacetylase (HDAC) inhibitors' that can activate the expression of methylated genes by increasing the accessibility of the demethylase enzyme to the DNA. ${ }^{16,1715,16}$, A trial of VPA treatment in an ovine model of McArdle disease

Formatted: Superscript resulted in an increased number of glycogen phosphorylase positive skeletal muscle fibres, suggesting activation of BGP. ${ }^{18}-17$ Encouraging results were also obtained in an in vitro $\ominus$ knock-in (KI) mouse model of McArdle disease carrying the p.R50X mutation. Following VPA exposure, cultured myotubes from the mouse model expressed BGP in association with a dose-dependent decrease in muscle glycogen accumulation. ${ }^{17} \underline{18}$

Based on this preclinical research, VPA could be considered as a potential therapeutic target for McArdle disease. This study was designed as a feasibility/pilot study to: a) determine whether or not VPA has an effect on BGP expression and b) to power a future randomised, placebo-controlled study (RCT).

\section{z. METHODS:}

The study was conducted at two sites: UCL Institute of Neurology, London, UK and Rigshospitalet, Copenhagen, Denmark. Protocol and study documents were

Formatted: Font: (Default) Arial, Bold Formatted: Normal, No bullets or numbering 
approved by ethical committees and regulatory bodies for each site. Informed consent was obtained from all participants prior to any study procedures and the study was conducted in line with good clinical practice as determined by the Declaration of Helsinki. The trial was registered at ClinicalTrials.gov (NCT03112889).

\subsection{Study design}

A phase II, open label, multi-centre feasibility study.

\subsection{Participants}

2.2.1. Based upon previous research in McArdle disease, it was anticipated that

data from 16 participants would be adequate to provide a good estimate of the standard deviation of the change in the $\mathrm{VO}_{2}$ peak to inform the sample size calculation for a future RCT. ${ }^{19}$

All participants had a genetically confirmed diagnosis of McArdle disease and were over 18 years of age.

Based upon previous research in McArdle disease, it was anticipated that data from

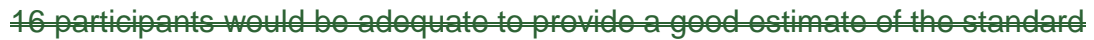

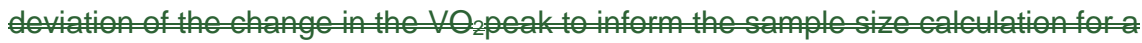
future ROT ${ }^{19}$

Inclusion criteria:

All participants were over 18 years of age and diagnosed with GSDV (confirmed by DNA analysis for recessive mutations in PYGM and/or muscle biopsy showing subsarcolemmal blebs of glycogen and absence of skeletal muscle glycogen phosphorylase on histochemical stain. All participants (male and female) $\underline{\text { had to use contraception throughout the study unless they were post-menopausal or }}$ infertile.
Formatted: Font: (Default) Arial, Bold, Font color: Text 1 Formatted: Font: (Default) Arial, Font color: Text 1 Formatted: Normal, No bullets or numbering

Formatted: Font: (Default) Arial, Bold, Font color: Text 1 Formatted: Normal, No bullets or numbering Formatted: Font: (Default) Arial

Formatted: Font: (Default) Arial, Superscript

Formatted: Font: (Default) Arial, Font color: Text 1, English (United Kingdom)

Formatted: Indent: Left: $0.5^{\prime \prime}$

Formatted: Font: (Default) Arial

Formatted: Normal, No bullets or numbering

Formatted: Line spacing: Double, No bullets or numbering

Formatted: Font: (Default) Arial, 12 pt

Formatted: Font: (Default) Arial, $12 \mathrm{pt}$

Formatted: Font: Italic

Formatted: Font: (Default) Arial, $12 \mathrm{pt}$

Formatted: Font: (Default) Arial, $12 \mathrm{pt}$

Formatted: Font: (Default) Arial, $12 \mathrm{pt}$ 
2.2.2.

All participants were required to use contraceptives for the duration of the study unless they were clinically confirmed infertile or post-menopausal women. Participants had to have a normal acyl-carnitine profile at screening and had to be able to perform the exercise assessments.

\section{Exclusion criteria:}

2.2.3. The following were exclusion criteria: pregnancy, diabetes, inflammatory disorders e.g.systemic lupus erythematosus, sensitivity/allergy to VPA, treatment with VPA within 12 months prior to recruitment, pre-existing liver disease or a family $\underline{\text { history of severe liver disease affecting a first degree relative, anti-convulsant }}$ medication or any other medication known to interact with VPA, sensitivity to local anaesthetics that would prevent muscle biopsy, any co-morbid illness or disability which would prevent an exercise assessment. Other metabolic condition affecting either the patient or a first-degree relative such as porphyria, mitochondrial disease, abnormal acyl carnitine profle or low serum carnitine

Participants with a history of diabetes, inflammatory disease, liver disease, porphyria or positive family history of either liver or mitochondrial disease were excluded. A list of inclusion and exclusion criteria can be found at

ClinicalTrials.gov (NCT03112889).in Supplement $x x_{\text {is }}$

2.3. Intervention

All participants received VPA extended release tablets (by Sanofi-Aventis) once

daily. Participants were warned of the expected VPA related side-effects such as weight gain, fatigue, alterations in blood indices and risk of liver damage. VPA was introduced slowly with an escalating dose regimen with $5 \mathrm{mg} / \mathrm{kg} /$ day increments each week for three weeks up to the full dose treatment $(20 \mathrm{mg} / \mathrm{kg} /$ day $)$. This dose was
Formatted: Font: (Default) Arial, Underline, Font color: Text 1

Formatted: Normal, No bullets or numbering

Formatted: Font: (Default) Arial, English (United Kingdom) Formatted: Indent: Left: $0.5^{\prime \prime}$

Formatted: Font: (Default) Arial

Formatted: Normal, No bullets or numbering

Formatted: Font: (Default) Arial, English (United Kingdom)

Formatted: Font: (Default) Arial, English (United Kingdom)

Formatted: Default, Space After: $13.6 \mathrm{pt}$, No bullets or numbering

Formatted: Font: (Default) Arial, English (United Kingdom)

Formatted: Font: (Default) Arial, English (United Kingdom)

Formatted: Font: (Default) Arial, English (United Kingdom)

Formatted: Font: (Default) Arial, English (United Kingdom)

Formatted: Font: (Default) Arial, English (United Kingdom)

Formatted: Font: (Default) Arial, English (United Kingdom)

Formatted: Font: (Default) Arial, English (United Kingdom)

Formatted: Font: (Default) Arial, English (United Kingdom)

Formatted: Font: (Default) Arial, English (United Kingdom)

Formatted: Font: (Default) Arial

Formatted: Font: (Default) Arial, English (United Kingdom)

Formatted: Indent: Left: $0.25 "$

Commented [nn3]: Renata to update the Supplement list prior to resubmission

Formatted: Highlight

Formatted: Font: $12 \mathrm{pt}$, Highlight

Formatted: Highlight

Formatted: Font: (Default) Arial, English (United Kingdom)

Formatted: Font: (Default) Arial

Formatted: Normal, Line spacing: single, No bullets or numbering 
chosen as it is the lowest recommended dose for treating epilepsy and we wanted to minimise known drug-related side-effects such as weight gain, drowsiness and thrombocytopenia that might outweigh any potential benefit. The daily dose was rounded up to the nearest available tablet strength and the maximum dose was $2 \mathrm{~g} /$ day. The ovine clinical trial conducted over 15 weeks showed an increase in the $\underline{\text { number of phosphorylase positive fibres over time }{ }^{17} \text {. We decided to treat our patients }}$ for 6 months, a longer time-period than the ovine trial, to maximise any potential positive impact. After six months on full dose treatment and after the final study visit, VPA dose was reduced by $5 \mathrm{mg} / \mathrm{kg} /$ day each week for three weeks and then discontinued.

Dose selection: The $20 \mathrm{mg} / \mathrm{kg} /$ day dose was selected based on the evidence available for VPA use as a treatment option for epilepsy (NICE, 2018). The suggested maintenance dose for epilepsy treatment is 20-30 mg/kg/day (NICE)

2018). Thus the lowest recommended therapeutic dose for epilepsy was chosen.

Io reduce the risks of having a negative trial due to short treatment duration, a sixmonth treatment period was selected; slightly longer than the treatment period used in the ovine model study.

\subsubsection{Study visits}

At screening, participants underwent a full medical history and examination. Investigations included ECG, laboratory blood tests for free carnitine, acyl carnitine profile, full blood count, liver and renal function. Participants performed an incremental baseline cycle test to determine exercise capacity.

Following screening, there were three study visits at week 0 (baseline - V1), week 16 (+-7 days - V2) and week 28 (+-7 days - V3). In between visits participants were 
telephoned every 4 weeks (+- 7 days) from baseline until week 40 to assess adverse events (AEs) and study compliance.

Assessment of Compliance. Compliance was also assessed at each study visit ${ }_{2}$ and during telephone calls and returned pills were counted on V2 and V3. Compliance $>90 \%$ was the minimum threshold for participants to continue in the trial.

\subsection{Outcome measures}

Screening visit cycle test: All participants exercisedeycled on a cycle ergometer.

Oxygen consumption was assessed with the Cortex ergospirometry system (Cortex Metalyzer II, Cortex Biophysik GmbH, Leipzig, Germany) in the UK or Quark CPET (Cosmed Srl., Milan, Italy) in DK. An incremental cycle ergometer test was performed (from zero to $20 \mathrm{~W}$ in the first minute, increased by at least $5 \mathrm{~W}$ every two minutes) to determine each participant's aerobic capacity $\left(\mathrm{VO}_{2}\right.$ peak).

\section{Primary outcome:}

2.5.1. $\mathrm{VO}_{2}$ peak was -measured in a constant-to-maximal workload cycle test on $\mathrm{V} 1$, V2 and V3. After fasting for four hours, participants cycled for 15 minutes at a constant workload at $65 \%$ of the $\mathrm{VO}_{2}$ peak determined in the screening test. After 15min, the power output was increased by at least five watts every minute until maximal volition to determine the $\mathrm{VO}_{2}$ peak.

\subsubsection{Secondary outcomes;}

a) Muscle biopsy to assess number of phosphorylase positive fibres: V1 and V3. Where available, recent diagnostic muscle biopsies of good quality undertaken prior to screening were used for analysis at baseline. The presence of phosphorylase was assessed using histochemistry and neonatal myosin staining was used to assess the presence of possible regenerating
Formatted: Font: (Default) Arial, Bold, Font color: Text 1

Formatted: Normal, No bullets or numbering

Formatted: Font: (Default) Arial, Font color: Text 1

Formatted: Normal, No bullets or numbering

Formatted: Font: (Default) Arial, Font color: Text 1

Formatted: Font: (Default) Arial, Font color: Text 1 
fibres. The histochemical method used identifies all isoforms of

\section{phosphorylase, given that the mutations in PYGM result in no phosphorylase}

expression, it was not considered necessary to include other methods to

\section{identify specific phosphorylase isoforms such as PCR. Muscle biopsy slides}

were scanned, and the number of glycogen phosphorylase muscle fibres were counted using ImageJ imaging software.

b) Plasma lactate levels during a non-ischaemic forearm test: V1, V2, V3.

Repetitive maximal handgrip contractions using a hand-held dynamometer were performed every other second for one minute. Plasma lactate and serum ammonia levels were analysed at 0, 2 and 5 minutes.

c) 12-Minute Walk Distance (12MWD): V1, V2, V3 after 45 minutes of rest, following the cycle ergometer test. Participants were required to complete as many $10 \mathrm{~m}$ shuttle walks as possible for 12 minutes on a marked corridor. ${ }^{20}$ The total walked distance was analysed.

d) Quality of life assessment: Short Form 36 (SF36 health survey) V1, V2, V3 was completed and scored using the QualityMetric Health Outcomes ${ }^{\mathrm{TM}}$ Scoring Software. Normative values of the 2009 population graded by age and gender:

e) Safety measures: All participants completed a symptom diary, which included: concomitant medications use, adverse events, myoglobinuria and significant worsening of McArdle symptoms information. The study team recorded adverse events at each visit and at frequent telephone calls. During V1, V2 and V3 safety blood analyses included: full blood count, CK, LFT, U\&E, platelets, coagulation screen (PT, APTT, INR and fibrinogen), lactate, ammonia, glucose and VPA blood level.
Formatted: Font: Italic

Formatted: Font: (Default) Arial, 12 pt, Font color: Text 1 


\section{f) Adverse events were assessed.}

\subsection{Statistical analysis}

Due to the pilot nature of the study, the study was not powered to show statistically significant differences between treatment groups, and so all analysis was descriptive in nature. Summaries at each time point were produced, in addition to summaries of the changes from baseline to both V2 and V3. Changes from baseline were also calculated as a percentage of the baseline. Continuous variables were summarised by the mean and standard deviation and data range if found to follow a Normal distribution, and by the median and inter-quartile range, and data range if not normally distributed. Categorical variables were summarised by the frequency and percentage of values in each category.

The primary outcome was $\mathrm{VO}_{2}$ peak, measured during exercise on a cycle ergometer at maximum volition. Clinically important increases in $\mathrm{VO}_{2}$ peak and 12-minute walk distance were predefined defined as greater than $10 \%$ of the baseline value. The clinical importance of any effects was compared to this fixed value.

We planned to use these data to provide an estimate of standard deviation of the change in each of these factors that would be required for the sample size calculation of a larger RCT in the future. Since exercise capacity in McArdle disease is relatively stable over time it was anticipated that baseline data from this study and pooled data from previously published studies would be able to provide data for the placebo arm of a future RCT.

\section{RESULTS:}

Formatted: Font: (Default) Arial, Bold

Formatted: Font: (Default) Arial

Formatted: Font: (Default) Arial, Bold

Formatted: Normal, No bullets or numbering
Formatted: Normal, No bullets or numbering

Formatted: Font: (Default) Arial, Bold 


\subsection{Participants demographics.}

19 participants were screened, and 17 recruited (1느 men and 5 women), mean age was 46.2 years (range 21 to 67 years). One recruited participant was withdrawn following screening because he failed to attend a pre-treatment muscle biopsy and V1 assessments (baseline). Two participants failed screening as they did not meet inclusion/exclusion criteria. Two participants dropped out between V1 and V2: one was lost to follow up and the second dropped out because of gastrointestinal AEs. In total, 16 participants attended $\mathrm{V} 1$ while 14 participants completed all trial visits. Mean drug compliance at V3 was $98.7 \% \pm 1.6$ (range: $95-100)$. The mean VPA level at V2 was 72+-27 (range 29-132) and at V3 66 +-23 (range 28-101). Safety blood analyses did not demonstrate any clinically significant alterations.

\subsection{Primary outcome:}

There was no improvement in $\mathrm{VO}_{2}$ peak from baseline to $\mathrm{V} 3$ measured by the cycle test (Figure 1, Table 1).

\subsection{Secondary Outcomes:}

Exercise testing: Results for the secondary outcome measures associated with the cycle test are shown in table 2 . There was no clinically meaningful change between baseline and V3.

Muscle biopsy analysis: The median percentage change in the number of phosphorylase positive fibres fromat baseline and V3 was $\underline{0.0 z e r o}(\operatorname{IQR} 0.0,0.2)$, while the mean percentage change in the number of of neonatal myosin positiveregenerating fibres was $0.6+/-2.2-2.1$ at V3 (SD: \pm 2.9$)$ but these fibres did not indicating no-expression of phosphorylase.ase enzyme in skeletal muscle after treatment.
Formatted: Font: (Default) Arial, Bold, Font color: Text 1

Formatted: Font: (Default) Arial, Bold, Font color: Text 1

Formatted: Normal, No bullets or numbering 
Forearm exercise test: The mean +/-SD change in plasma lactate from baseline to V3 was $0.12+/-0.34$ (range $-0.52,0.78$ ) indicating no clinically meaningful difference.

12-minute walk test: The mean total distance walked was 966m (range 683-1292m) at baseline and $949 \mathrm{~m}$ (range $606-1690 \mathrm{~m}$ ) at V3.The mean variation in the total walked distance was $31 \mathrm{~m}$ from baseline to visit 3 was $31 \mathrm{~m}$, indicating a $3 \%$ change which does not represent a clinically meaningful difference.

Quality of life: Results for the SF36 Quality of life questionnaire are shown in table 3. There were no clinically meaningful changes in the two main SF36 health domain scales from baseline to V3.

\section{Adverse Events}

VPA was well tolerated well. None of the participants experienced myoglobinuria during the course of the trial. Table 4 summarises AE data. There were a mean of 10 adverse events per participant (155 in total), most were rated as mild (67\%) and unrelated to the study drug $(60 \%)$. Weight gain was the only definite drug-related $\mathrm{AE}$, the mean weight gain from $\mathrm{V} 1$ to $\mathrm{V} 3$ was $+3.5 \mathrm{~kg}$ (SD: 4.8 ; range: $-3 \mathrm{~kg}$ to $+17 \mathrm{~kg}$ ) considered to be within the expected range for individuals taking VPA for other reasons. There were $21 \mathrm{AEs}$ (14\%) deemed as 'probably' related to VPA. There was one SAE, which was not considered to have been related to the study drug. One participant withdrew from the study due to gastrointestinal symptoms considered to have been related to VPA use.

\section{DISCUSSION:}


primary endpoint: change in $\mathrm{VO}_{2}$ peak, and secondary endpoints: total distance walked on a 12MWT, forearm exercise, increase in lactate production test, histochemicalfollowing exercise, the expression of phosphorylase enzyme in skeletal muscle and safety blood parameters. There was no clinically meaningful change from baseline to visit three for any of the primary and secondary endpoints. and changes in total walked distances. All assessed endpoints failed to confirm benefits of VPA in the assessed sample.

VPA has previously been shown to stimulate the brain isoform of phosphorylase-expression in two animal models of McArdle disease ${ }^{17,18}$. In the sheep model, animals received increasing doses of enteric administration of enteral VPA (20 - 60mg/kg body weight). Muscle biopsies were performed at different times during the treatment phase, and in different muscle groups. In the same study, a group of sheep received intramuscular injections of VPA. An increase in phosphorylase positive fibres was seen in post-treatment muscle biopsies, which increased with higher doses of VPA and over time. However, neonatal myosin staining was not reported to confirm if the phosphorylase activity waspositive fibres Formatted: Not Highlight were related to mature muscle fibres or to regenerating fibres or induced. Although neonatal myosin is often used as a marker for fibre regeneration there is evidence it $\underline{\text { can be up-regulated }}{ }^{20}$. Muscle biopsies from a variety of neuromuscular conditions often show fibres with neonatal myosin for unknown reasons ${ }^{14}$. In our study, the presence of a few fibres that showed neonatal myosin but no phosphorylase suggests that these were not regenerating fibres but rather that neonatal myosin had been upregulated. There are currently no antibodies to the brain isoform that reliably work on human muscle biopsies, however, the histochemical stain for phosphorylase 
detects all three isoforms of phosphorylase, we are therefore confident that VPA treatment did not up-regulate either the brain or liver isoform in our participants.

In the earlier sheep study ${ }^{17}$, it wais not possible to completely exclude a local toxic effect of intramuscular IM-VPA, which could have triggered muscle regeneration and thus the expression of foetal isozyme in injected muscles. Saline injected sheep showed a few fibres with neonatal myosin suggesting that mild muscle damage may have resulted from injection. In addition, there was a mild inflammatory response, which was not seen in the muscle biopsies of participants in this trial who were treated with oral VPA. However, aHowever, an in vitro study analysed muscle cultures from KI mice exposed to VPA for 72 hours at 1,2 and 5 $\mathrm{mM}^{17}$ and showed a. A dose-dependent increase in BGP was shown together with a reduction in intracellular glycogen content 17.18

VPA is a well-known drug prescribed as a treatment option for epilepsy and migraine ${ }^{21}$ (Linde et al., 2013). Its efficacy has also been evaluated for other conditions, including bipolar disorders and schizophrenia, ${ }^{22,23}$ (Wang et al., 2016, Cipriani et al., 2013). More recently, studies in the field of neuromuscular disease have explored the role of VPA as a histone deacetylase inhibitor (HDAC). Even though in vitro studies have indicated an beneficial-effect of VPA in spinal muscular atrophy, similar efficacy was nothas not been confirmed in clinical trials 24,25,26by clinicat trials performed in humans with the condition (Kisselet al., 2014, Krosschellet al., 2018, Kisselet al., 2011). A Phase III study of VPA in amyotrophic lateral sclerosis showed no evidence for slowingalso failed to demonstrate effectiveness in disease progression or increasing and-surviva| ${ }^{27}$ t. (Piepers et al., 2009). A recent study suggested that VPA may be of potential value in oculopharyngeal muscular dystrophy, but there has been no clinical trial in humans with the condition (Abu-Baker et al., 2018). 
It was not possible to predict the effect of VPA in humans with McArdle disease based on previous research into other neuromuscular conditions because they have different pathophysiologies. However, for McArdle disease, both in vitre and in vivo research in animal models has provided strong evidence that VPA had a beneficial effect, supporting further study of VPA in humans affected by the condition. Therefore, a proof-of-concept study was developed to confirm early VPA efficacy data in humans living with the condition.

This study confirmed VPA was safe in the assessed patient population. Weight gain, an expected VPA-related AE was also seen in McArdle disease patients. Even though VPA therapy was safe in the assessed patients, Tt This study showed weight gain and Gl disturbances to be the most significant side-effects from $\underline{V P A}$, otherwise the drug was well-tolerated, indicating its use would be safe for other indications, such as epilepsy. Migraine and bipolar disorder in the McArdle population.efficacy However, this small open label study failed to show any clinically meaningful therapeutic effect. results of this open label feasibility study did not confirm the findings from animal studies and no clinically meaningful benefit was found. Therefore, we do not recommend any further study of VPA in people with McArdle disease. However, the strategy to upregulate BGP as a possible therapeutic strategy may still be feasible using alternative HDAC agents in future trials.

\section{CONCLUSIONS:}

This feasibility study to assess efficacy of $20 \mathrm{mg} / \mathrm{kg} /$ day VPA in McArdle disease was planned to power a larger placebo-controlled trial of VPA in this patient population. Our results demonstrated that VPA was well-tolerated but there was no clinically meaningful benefitbenefit from baseline after 6 months of_VPA-treatment.
Commented [nn10]: Reviewer: Was the weight gain more than reported in cohorts of patients with epilepsy treated with VPA? Were patients warned for this?

would not compare with epilepsy, the background is differen (eg some patients have epilepsy and other conditions with cognitive impairment) or use additional anti-epileptic drugs in combination. I would not combine both as theyare completely different populations (epilepsy $x$ NMD field)

Commented [nn11]: Reviewer: Did the results in this study lead to any other new insights?

Commented [nn12]: Reviewer: It would be nice if the authors could discuss the reasons why they think that VPA did not exert the expected effect in some more detail

I can generate a paragraph on why VPA failed in other diseases. Please guide me on the strategy and I develop the argument

Commented [nn13]: Reviewer: Could the authors add which alternative HDAC agents could be used in future trials?

Do we think VPA did not work per se, ot

Formatted: Font: (Default) Arial, Bold

Formatted: Normal, No bullets or numbering 


\section{Acknowledgments:}

\section{We would like to dedicate this article to the memory of Professor John Howell who}

\section{pioneered this work in an ovine McArdle disease model.}

We would like to thank Muscular Dystrophy UK for funding this study and AGSDUK for their support in recruitment. This research was supported by the National Institute for Health Research University College London Hospitals Biomedical Research Centre. RSS received a scholarship from CAPES Foundation Ministry of Education Brazil, and the research study is described in more details in her PhD thesis. We would also like to acknowledge Professor John Howell, Professor Byron Kakuluss for hisand Professor Caroline Sewry for their advice and support during the development of the study protocol.

\section{Conflicts of interest:}

None of the authors report any conflicts of interest related to the study medication.

\section{REFERENCES}

1. Mc AB. Myopathy due to a defect in muscle glycogen breakdown. Clinical science 1951;10:13-35.

2. Mommaerts WF, Illingworth B, Pearson CM, Guillory RJ, Seraydarian K. A FUNCTIONAL DISORDER OF MUSCLE ASSOCIATED WITH THE ABSENCE OF PHOSPHORYLASE. Proceedings of the National Academy of Sciences of the United States of America 1959;45:791-797.

3. Schmid R, Mahler R. Chronic progressive myopathy with myoglobinuria: demonstration of a glycogenolytic defect in the muscle. The Journal of clinical investigation 1959;38:2044-2058. 
4. Andreu AL, Nogales-Gadea G, Cassandrini D, Arenas J, Bruno C. McArdle disease: molecular genetic update. Acta myologica : myopathies and cardiomyopathies : official journal of the Mediterranean Society of Myology / edited by the Gaetano Conte Academy for the study of striated muscle diseases 2007;26:53-57.

5. Scalco RS, Chatfield S, Junejo MH, Booth S, Pattni J, Godfrey R, et al. McArdle Disease Misdiagnosed as Meningitis. The American journal of case reports $2016 ; 17: 905-908$.

56.Genes and exercise intolerance: insights from McArdle disease. G NogalesGades, R Godfrey, J Coll-Conti, G Pintos-Morell, T Pinos, J Arenas, MA martin, A Lucia. Physiol. Genomics 2016;48: 93-100 Martin MA, Lucia A, Arenas J, Andreu AL. Glycogen Storage Disease Type V. In: Pagon RA, Adam MP, Ardinger HH, et al., eds. GeneReviews(R). Seattle (WA): University of Washington, Seattle University of Washington, Seattle. GeneReviews is a registered trademark of the University of Washington, Seattle. All rights reserved., 1993.

7. Brady S, Godfrey R, Scalco RS, Quinlivan RM. Emotionally-intense situations can result in rhabdomyolysis in McArdle disease. BMJ case reports 2014;2014.

68. Santalla A, Nogales-Gadea G, Encinar AB, Vieitez I, Gonzalez-Quintana A, Serrano-Lorenzo P, et al. Genotypic and phenotypic features of all Spanish patients with McArdle disease: a 2016 update. BMC genomics 2017;18:819.

79. Quinlivan R, Martinuzzi A, Schoser B. Pharmacological and nutritional treatment for McArdle disease (Glycogen Storage Disease type V). The Cochrane database of systematic reviews 2014;11:Cd003458.

810. Crerar MM, Karlsson O, Fletterick RJ, Hwang PK. Chimeric muscle and brain glycogen phosphorylases define protein domains governing isozyme-specific 
responses to allosteric activation. The Journal of biological chemistry 1995;270:13748-13756.

911. Bartram C, Edwards RH, Beynon RJ. McArdle's disease-muscle glycogen phosphorylase deficiency. Biochimica et biophysica acta 1995;1272:1-13.

1012. Newgard CB, Hwang PK, Fletterick RJ. The family of glycogen phosphorylases: structure and function. Critical reviews in biochemistry and molecular biology 1989;24:69-99.

113. Sato K, Imai F, Hatayama I, Roelofs RI. Characterization of glycogen phosphorylase isoenzymes present in cultured skeletal muscle from patients with McArdle's disease. Biochemical and biophysical research communications 1977;78:663-668.

124. DiMauro S, Arnold S, Miranda A, Rowland LP. McArdle disease: the mysterious appearance of phosphorylase activity in cells that ought to lack the genetic program. A fetal isoenzyme? Transactions of the American Neurological Association 1977;102:112-115.

135. Martinuzzi A, Schievano G, Nascimbeni A, Fanin M. McArdle's disease. The unsolved mystery of the reappearing enzyme. The American journal of pathology 1999;154:1893-1897.

\section{4. $\forall$ Dubowitz, G Sewry CA, A Oldfors A. Metabolic Myopathies 1: Glycogenoses}

\section{and Lysosomal Myopathies. In: Auscle Biopsy: A Practical Approach. Fifth Edition}

\section{0, Elsevier Oxford}

156. Brodie SA, Brandes JC. Could valproic acid be an effective anticancer agent? The evidence so far. Expert review of anticancer therapy 2014;14:1097-1100. 167. de Luna N, Brull A, Guiu JM, Lucia A, Martin MA, Arenas J, et al. Sodium valproate increases the brain isoform of glycogen phosphorylase: looking for a 
compensation mechanism in McArdle disease using a mouse primary skeletalmuscle culture in vitro. Disease models \& mechanisms 2015;8:467-472.

178. Howell JM, Dunton E, Creed KE, Quinlivan R, Sewry C. Investigating sodium valproate as a treatment for McArdle disease in sheep. Neuromuscul Disord

Formatted: Portuguese (Brazil) 2015;25:111--19.

18.N De Luna, A Brull, J Guiu, A lucia, MA Martin, J Arenas, R Marti, A andreu, T Pinos. disease models and mechanisms 2015;8 467-72

19. Julios S, Sample size of 12 per group rule of thumb for a pilot study.

Pharmaceutical Statistics 2005; 4: 287-291.

20. AD Grounds MD. The need to more precisely define aspects of skeletal muscle regeneration. Int J Biochem 2014; 56: 56-65

21. Linde, M., Mulleners, W. M., Chronicle, E. P.McCrory, D. C. 2013. Valproate

(valproic acid or sodium valproate or a combination of the two) for the prophylaxis of episodic migraine in adults. Cochrane Database Syst Rev, Cd010611.

22. Wang, Y., X, J., Helfer, B., LI, C. \& Leucht, S. 2016. Valproate for schizophrenia.

Cochrane Database Syst Rev, 11, Cd004028.

23 Cipriani, A., Reid, K., Young, A. H., Macritchie, K. Geddes, J. 2013. Valproic acid, valproate and divalproex in the maintenance treatment of bipolar disorder. Cochrane Database Syst Rev, Cd003196

24. Kissel, J. T., Elsheikh, B., King, W. M.et al,2014. SMA valiant trial: a prospective, double-blind, placebo-controlled trial of valproic acid in ambulatory adults with spinal muscular atrophy. Muscle Nerve, 49, 187-92. 
25. Krosschell, K. J., Kissel, J. T., Townsend, E. L., et al2018. Clinical trial of L-

Carnitine and valproic acid in spinal muscular atrophy type I. Muscle Nerve, 57, 193-

$\underline{99}$

26. Kissel, J. T., Scott, C. B., Reyna, S. P., J. et al,2011. SMA CARNIVAL TRIAL

PART II: a prospective, single-armed trial of L-carnitine and valproic acid in

ambulatory children with spinal muscular atrophy. PLoS One, 6, e21296.

27. Piepers, S., Veldink, J. H., De Jong, S. W.,et al,2009. Randomized sequential trial

of valproic acid in amyotrophic lateral sclerosis. Ann Neurol, 66, 227-34.
Formatted: Font: (Default) Arial, Not Highlight

Formatted: Font: (Default) Arial, Not Highlight

Formatted: French (Switzerland)

Formatted: Font: (Default) Arial, Not Highlight

Formatted: Font: (Default) Arial, Not Highlight

Formatted: French (Switzerland)

Formatted: Font: $12 \mathrm{pt}$

Formatted: Font: $12 \mathrm{pt}$, Not Highlight

Formatted: Font: $12 \mathrm{pt}$, Not Highlight

Formatted: Font: $12 \mathrm{pt}$

Formatted: French (Switzerland)

Formatted: French (Switzerland) 
Figure 1

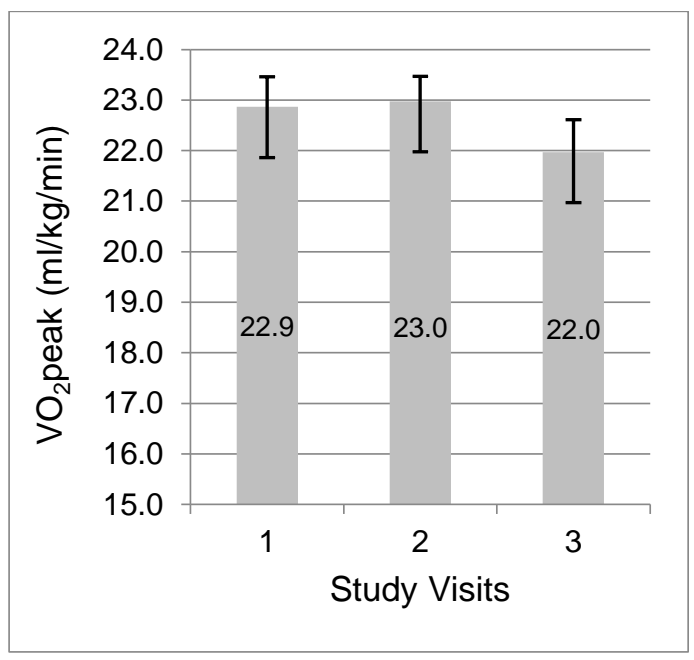

Mean $\mathrm{VO}_{2}$ peak for participants who completed all trial visits $(n=14)$. Values are mean \pm standard deviation. On V2: two participants were not included in mean $\mathrm{VO}_{2}$ peak analysis as they did not perform a maximal test. 
Click here to access/download

Table

Table 1.docx 
Table 2

Click here to access/download

Table

Table 2.docx 
Table 3

Click here to access/download

Table

Table 3.docx 
Table 4

Click here to access/download

Table

Table 4.docx 
The corresponding author ensures all co-authors have read and agreed the contents of the paper 